\title{
Effect of an Alternative Newborn Naming Strategy on Wrong-Patient Errors: A Quasi-Experimental Study
}

\author{
Ethan Pfeifer ${ }^{1,2}$ Margaret Lozovatsky ${ }^{3}$ Joanna Abraham ${ }^{1,2}$ Thomas Kannampallil ${ }^{1,2}$ \\ ${ }^{1}$ Department of Anesthesiology, Washington University School of \\ Medicine, St Louis, Missouri, United States \\ Address for correspondence Thomas Kannampallil, PhD, Department \\ of Anesthesiology, School of Medicine, Washington University in \\ 2 Institute for Informatics, Washington University School of Medicine, \\ St Louis, Missouri, United States \\ St Louis, 4990 Children's Place, St Louis, MO 63110, United States
}

${ }^{3}$ Department of Pediatrics, Washington University School of

Medicine, St Louis, Missouri, United States

Appl Clin Inform 2020;11:235-241.

\section{Abstract}

Keywords

- wrong-patient errors

- newborn naming

- segmented regression

- patient misidentification
Objectives Newborns are often assigned temporary names at birth. Temporary newborn names-often a combination of the mother's last name and the newborn's gender-are vulnerable to patient misidentification due to similarities with other newborns or between a mother and her newborn. We developed and implemented an alternative distinct naming strategy, and then compared its effectiveness on reducing the number of wrong-patient orders with the standard distinct naming strategy.

Methods This study was conducted over a 14-month period in the newborn nursery and neonatal intensive care units of three hospitals that were part of the same health care system. We used a quasi-experimental study design using interrupted time series analysis to compare the differences in wrong-patient orders (an indicator of patient misidentification) before and after the implementation of the alternative distinct naming strategy.

Results Overall, there were 25 wrong-patient errors per 10,000 orders during entire study period (36.8 per 10,000 before and 19.6 per 10,000 after). However, there was no statistically significant change in the rate of wrong-patient ordering errors after the transition from the distinct to the alternative distinct naming strategy $(\beta=0.832,95 \%$ confidence interval $[\mathrm{Cl}]=-0.83$ to $2.49, p=0.326$ ). We also found that, overall, $1.7 \%$ of the clinicians contributed to $62 \%$ of the wrong-patient errors.

Conclusion Although we did not find statistically significant differences in wrongpatient errors, the alternative distinct naming approach provides pragmatic advantages over its predecessors. In addition, the localization of wrong-patient errors within a small set of clinicians highlights the potential for developing strategies for delivering training to clinicians.

\section{Background and Significance}

Nearly $12 \%$ of over 4 million newborns in the United States are admitted to a neonatal intensive care unit (NICU). ${ }^{1}$ A significant patient-safety threat in the NICU is patient misidentification, ${ }^{2}$ often arising from similarities in names or medical record numbers (MRN) among patients, ${ }^{3-5}$ and due to challenges of

received

August 21, 2019

accepted after revision

January 29, 2020 electronic health record (EHR) user interfaces in pediatric settings. ${ }^{6,7}$ Although patient misidentification has primarily been studied in the NICU, it is also prevalent in other settings such as newborn nurseries. ${ }^{8}$ Commonly attributed causes for patient misidentification are the use of temporary nondistinct names immediately after birth, MRNs being assigned
C 2020 Georg Thieme Verlag KG Stuttgart · New York
DOI https://doi.org/ 10.1055/s-0040-1705175. ISSN 1869-0327. 
sequentially, identical surnames for multiple patients, and multiple births (e.g., twins or triplets) with nearly identical names and MRNs. ${ }^{3-5,9}$ Strategies to prevent patient misidentification involve double checking of identifiers by clinicians such as MRN and date of birth. However, for newborns' with the same date of birth, sequentially assigned MRNs may be identical except for a single digit. Additionally, verbal confirmation of identity is also impossible with newborns.

Based on a national survey in the U.S. hospitals, Adelman and colleagues reported that nearly $82 \%$ of NICUs in the United States used a nondistinct naming strategy for temporary newborn names. ${ }^{9}$ Extending from this survey, they proposed and evaluated a distinct naming strategy for temporary newborn names. The distinct naming strategy had the following format: mother's first name, a possessive "s," newborn's gender and the mother's last name (e.g., the newborn of "Jane Doe" would be recorded as "Janesgirl Doe"). The distinct naming strategy decreased wrong-patient errors by $36 \%$ as compared with the nondistinct naming approach. ${ }^{5}$

To avoid patient misidentification errors, we developed and implemented an alternative distinct naming strategy within our institution's EHR. In this paper, we describe an exploratory study comparing the distinct naming strategy with the alternative distinct naming strategy to investigate its effects on patient misidentification (i.e., wrong-patient errors).

\section{Methods}

\section{Study Setting}

The study was conducted at three hospitals that are part of the BJC HealthCare system. Participating hospitals had an average annual number of deliveries of $850,1,608$, and 700 , respectively. The institutional review board of the university approved this study with a waiver of consent.

\section{Intervention: Alternative Distinct Naming Strategy}

The alternative distinct naming strategy seeks to address the problem of truncation of a patient's name in the header of their record. Truncations are problematic as they may cause the name of the mother and newborn to appear similar. As clinicians often have to simultaneously access both a newborn's chart and their mother's chart frequently, it increases the potential for erroneous actions (e.g., ordering a medication for the newborn instead of the mother).

In most EHRs, headers display patient names in the format "Last Name, First Name," but have limits on the number of characters that can be displayed. In the Epic EHR (Verona, Wisconsin, United States), there is a pixel limit on the header of approximately 158 pixels, allowing approximately 17 characters to be displayed. As characters have different pixel widths, the actual number of characters that can be displayed varies. As a result, when using the distinct newborn strategy, newborns with long last names can have their gender information cut-off and not show up on the header. For example, using the distinct naming strategy, "Washington, Marthasboy" will be displayed in the header as "Washington, Marth." The alternative distinct naming strategy would display the newborn's gender as a prefix to the mother's first name in the following manner: "Last Name, GenderFirst Name” (e.g., "Washington, BoyMartha”).

Differentiating the newborn's and the mother's names is a significant benefit of the alternative distinct naming strategy. In addition, the numbers present in the name of a multiple birth newborn will be replaced with the full word for that number (e.g., with the distinct naming strategy twins will be displayed as: "Doe, 1Janesgirl" and "Doe, 2Janesgirl"; with the alternative distinct strategy, these will be "Doe, OneGirlJane" and "Doe, TwoGirlJane"). This is likely to help to make these newborns easily distinguishable from each other, potentially avoiding wrong-patient orders. It must, however, be noted that even with the alternative distinct naming strategy, patients with very long last names can have the newborn's gender truncated (i.e., last name longer than approximately 17 characters). Each of the naming strategies is illustrated in -Fig. 1 .

\section{Outcome}

The primary outcome was the rate of retract-and-reorder events (RAR), an indicator for wrong-patient errors. A RAR event is defined as any order-medication, laboratory, imaging, or general care orders-placed and retracted on a patient within 10 minutes, and then reordered by the same clinician on a different patient within the next 10 minutes. RAR is also recommended by the National Quality Forum (NQF measure no.: 2723) and the Office of the National Coordinator for Health Information Technology ${ }^{10,11}$ as a metric for wrongpatient errors. The NQF determined that RAR events are "an outcome measure because while the error did not actually reach the patient, a wrong-patient retract and reorder in the EHR is still a medical error." The RAR measure was originally developed by Adelman and colleagues ${ }^{12}$ and was found to have a 76.2\% positive predictive value for identifying a wrong-patient order. RAR has been successfully used to measure the rate of intercepted wrong-patient errors in various settings including emergency departments, ${ }^{13,14}$ a children's hospital, ${ }^{12}$ and the NICU setting. ${ }^{4,5}$

\section{Study Design}

We used an interrupted time series (ITS) design to characterize the change in RAR event rate before and after the change in the naming strategy. ITS has been used extensively in quasi-experimental studies to evaluate the effects of EHR alerts $^{15,16}$ and other quality improvement efforts. ${ }^{17}$

\section{Data Collection}

We used a custom report that we developed to extract all orders placed via computerized clinician order entry and associated RAR events using the Epic EHR in the newborn nursery and the NICU at the considered hospitals within this period. The report included no identifiers of patients or clinicians. However, unique clinicians were represented with nonidentifiable sequential identification numbers (IDs).

All three hospitals made a change from the distinct naming strategy to the alternative distinct naming strategy on February 3, 2018. A RAR report was created for the time period between August 4, 2017 and September 30, 2018. 


\begin{tabular}{|c|c|c|c|c|}
\hline & Mother & $\begin{array}{l}\text { Neonate, } \\
\text { Non-Distinct Naming }\end{array}$ & $\begin{array}{l}\text { Neonate, } \\
\text { Distinct Naming }\end{array}$ & $\begin{array}{l}\text { Neonate, Alternate } \\
\text { Distinct Naming }\end{array}$ \\
\hline Header: & Doe, Jane & Doe, Babygirl & Doe, Janesgirl & Doe, Girljane \\
\hline Name: & Jane Doe & Babygirl Doe & Janesgirl Doe & Girljane Doe \\
\hline $\begin{array}{l}\text { EMR } \\
\text { Header: }\end{array}$ & Washington, Marth & Washington, Babyb & Washington, Marth & Washington, BoyMa \\
\hline Name: & Martha Washington & Babyboy Washington & $\begin{array}{l}\text { Marthasboy } \\
\text { Washington }\end{array}$ & $\begin{array}{l}\text { BoyMartha } \\
\text { Washington }\end{array}$ \\
\hline $\begin{array}{l}\text { EMR } \\
\text { Header: }\end{array}$ & LopezMartinez, Sa & LopezMartinez, Ba & LopezMartinez, Sa & LopezMartinez, Gi \\
\hline Name: & Sara LopezMartinez & $\begin{array}{l}\text { Babygirl } \\
\text { LopezMartinez }\end{array}$ & $\begin{array}{l}\text { Sarasgirl } \\
\text { LopezMartinez }\end{array}$ & $\begin{array}{l}\text { Girlsara } \\
\text { LopezMartinez }\end{array}$ \\
\hline $\begin{array}{l}\text { EMR } \\
\text { Header: }\end{array}$ & Balasubramanian, & Balasubramanian, & Balasubrumanian, & Balasubrumanian, \\
\hline Name: & $\begin{array}{l}\text { Divya } \\
\text { Balasubramanian }\end{array}$ & $\begin{array}{l}\text { Babyboy } \\
\text { Balasubramanian }\end{array}$ & $\begin{array}{l}\text { Divyasboy } \\
\text { Balasubramanian }\end{array}$ & $\begin{array}{l}\text { BoyDivya } \\
\text { Balasubramanian }\end{array}$ \\
\hline
\end{tabular}

Color key:

\begin{tabular}{|l|l|}
\hline $\begin{array}{l}\text { Newborn's name or header may be } \\
\text { similar to other newborns } \\
\text { (especially with a similar surname) }\end{array}$ & $\begin{array}{l}\text { Newborn's header is identical to } \\
\text { mother's header }\end{array}$ \\
\hline
\end{tabular}

Fig. 1 Three different strategies for temporary naming of newborns, with the appearance of their EMR tab headers shown above. The generic nondistinct naming, Adelman and colleagues' distinct naming, and the alternative naming strategy that we implemented. Yellow and red colored boxes indicate the failure of the naming system to provide a newborn name that is quickly distinguishable from the mother or other newborns. EMR, electronic medical record.

\section{Statistical Analysis}

For the purposes of analysis, we grouped orders and RAR events per week and computed the rate of RAR events per week (per 10,000 orders). We used a segmented quasiPoisson's regression (accounting for the extra dispersion) to investigate the changes in the overall rate of RAR events between the two naming strategy time periods. We used the segmented regression approach described by Wagner and colleagues. ${ }^{18}$ This model representing the segmented regression was constructed in the following manner:

$$
\begin{aligned}
Y_{\tau}= & \beta_{0}+\beta_{1} \times(\text { time })+\beta_{2} \times(\text { intervention }) \\
& +\beta_{3} \times(\text { time after intervention })+e_{\tau}
\end{aligned}
$$

where, $Y_{t}$ is the weekly rate of RAR events, time is a continuous variable indicating time in weeks (a total of 62 weeks), intervention is a categorical variable $(0,1)$ indicating the periods before ( 28 weeks) and after ( 34 weeks) the naming strategy change, and time after intervention is a variable indicating the number of weeks after the naming strategy change (sequential count,1-34). In this model, the intercept $\beta_{0}$ estimates the baseline rate of RAR events; $\beta_{1}$ estimates the overall secular trend of the rate of RAR events across the entire period; $\beta_{2}$ estimates the magnitude of the difference in the rate of RAR events immediately before and immediately after the naming change (i.e., representing the change in intercept); $\beta_{3}$ is the change in trend before and after the naming change (i.e., representing the change in slope). The error term $e_{t}$ represents the random variability not explained by the model at time $t$. It consists of an error term at time $t$ and a normally distributed random error.

In addition, we also identified clinicians (based on their IDs) with high rates of RAR events compared with their peers. For this, we used a one-tailed test of proportions where each clinician's rate of RAR events was compared against the pooled aggregate rate of all other clinicians. A one-tailed $t$-test was used, as we were only interested in identifying clinicians with RAR event rates higher than that of their peers. For each individual clinician, the two rates (i.e., individual clinician versus pooled aggregate of all other clinicians) were compared using a one-tailed test of proportions. The null hypothesis was that the individual clinician had a rate of RAR events that was not higher than the rest of the clinicians. Specifically, each individual clinician was compared against the pooled aggregate of all other clinicians, meaning that every individual clinician had their RAR event rate analyzed with a one-tailed $t$-test to see if it was higher than the pooled aggregate of all other clinicians' RAR event rate. A significance level of 0.05 was used unless otherwise specified. All analyses were conducted using R statistical software. ${ }^{19}$

As a secondary exploratory analysis of clinicians with higher RAR event rates, their orders and RAR events were further evaluated. To evaluate if a large number of orders were incorrectly placed for one patient, and then reordered for another patient, we examined whether there were multiple RAR events in a single week from a single provider for the same patient. This would represent a single error but might be overcounted as multiple RAR events. We also investigated the temporal distribution of RAR events.

\section{Results}

\section{Sample Characteristics}

Across the three hospitals, there were 30,105 orders over the study period (August 4, 2017-September 30, 2018) with a mean (M) of 494 orders/week with standard deviation (SD) 
Table 1 The time period, RAR event rate before and after naming strategy change

\begin{tabular}{|l|l|l|l|l|l|}
\hline Naming strategy & Dates & $\begin{array}{l}\text { No. of } \\
\text { weeks }\end{array}$ & $\begin{array}{l}\text { Mean orders/ } \\
\text { week (SD) }\end{array}$ & $\begin{array}{l}\text { Mean RAR events } \\
\text { per week (SD) }\end{array}$ & $\begin{array}{l}\text { RAR events per } \\
10,000 \text { orders }\end{array}$ \\
\hline Distinct naming & August 4, 2017-February 2, 2018 & 26 & $488(176)$ & $1.92(3.11)$ & 39.4 \\
\hline $\begin{array}{l}\text { Alternative } \\
\text { distinct naming }\end{array}$ & February 3, 2018-September 30, 2018 & 35 & $498(149)$ & $1.00(1.31)$ & 20.1 \\
\hline
\end{tabular}

Abbreviations: RAR, retract and reorder; SD, standard deviation.

of 160 (RAR event rate of 28.2 per 10,000 orders). There were a total of 1,913 patients in the study period. A total of 115 clinicians created orders during the study period.

\section{Rate of Retract-and-Reorder Events}

During the distinct naming strategy period, there were an average of 488 orders per week ( $S D=176)$, with a RAR event rate of 39.4 per 10,000 orders. During the alternative distinct naming strategy period, there was an average of 498 orders per week $(S D=149)$, with a RAR event rate of 20.1 per 10,000 orders. A summary of the order characteristics during each naming strategy period is shown in -Table 1.

Over the entire study period, we found a statistically significant negative secular trend $\left(\beta_{1}=-0.082,95 \%\right.$ confidence interval $[\mathrm{CI}]=-0.15$ to $-0.011, p=0.02)$. There was no statistically significant change in intercept $\left(\beta_{2}=0.832, \mathrm{CI}=\right.$ -0.83 to $2.49, p=0.326$ ) or slope of the rate of RAR events after the naming strategy change $\left(\beta_{3}=0.068, \mathrm{CI}=-0.02\right.$ to $0.16 ; p=0.134 ;$-Fig. 2).

\section{Clinician-Level Change in Rate of Retract-and-Reorder Events}

A total of 115 clinicians created orders during the study period. When error rates across the entire study period were analyzed, only $1.7 \%$ of clinicians ( 2 out of 115 ) were found to have significantly higher rate of RAR events than the rest of the clinicians $(p<0.05)$. These clinicians accounted for $62 \%$ of the total RAR events (53 out of 85 RAR events), while accounting for $17 \%$ of the total orders $(5,091$ of 30,105 orders). Overall, these clinicians had a RAR event rate of 104 per 10,000 orders, more than three times the overall rate (28 per 10,000 orders). During the distinct naming strategy period, these clinicians entered 2,749 orders (RAR event rate of 138 per 10,000 orders) and entered 2,342 orders (RAR event rate of 64 per 10,000 orders) during the alternate distinct naming period.

The secondary exploratory analysis of the RAR events of clinicians with higher RAR event rates showed no clinician had multiple RAR events for the same patient in a single

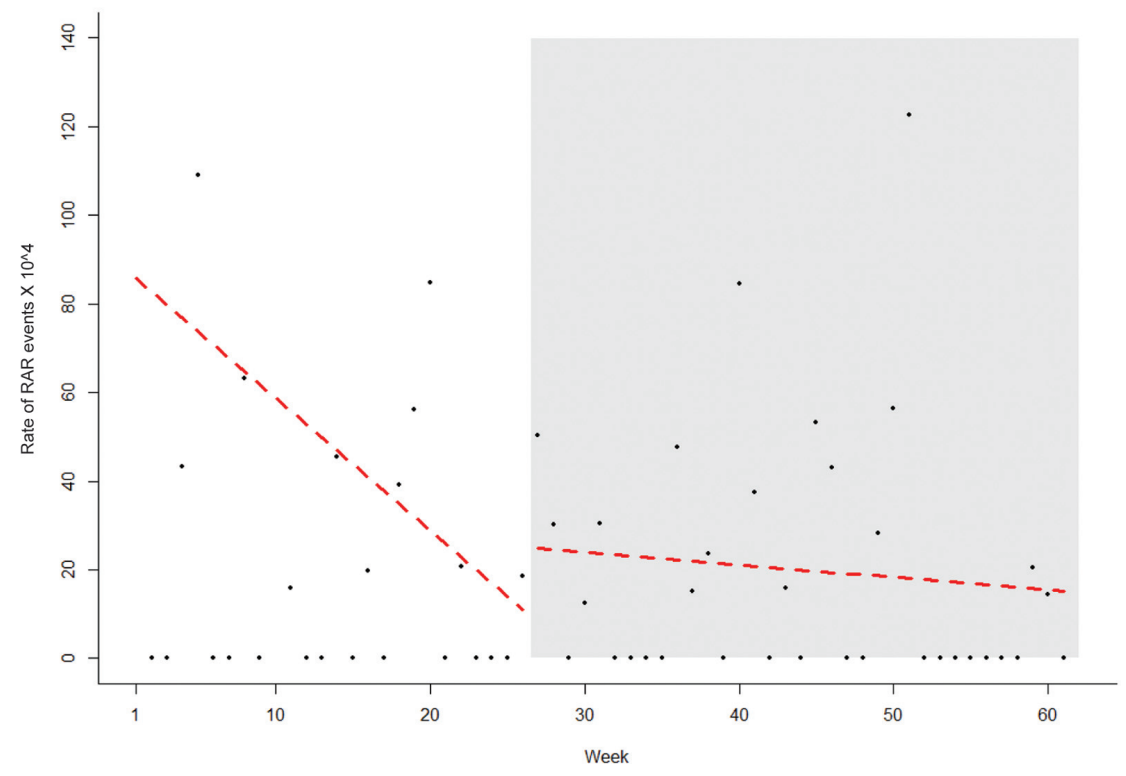

\begin{tabular}{|l|l|l|l|c|}
\hline & Estimate & Standard error & $\begin{array}{c}\text { Exponent } \\
(\mathbf{9 5 \%} \mathbf{C l})\end{array}$ & $p$-Value \\
\hline Intercept $\beta 0$ & 4.534 & 0.407 & 3.74 to 5.33 & - \\
\hline Original trend $\beta 1$ & -0.082 & 0.036 & -0.15 to -0.011 & 0.021 \\
\hline Change in intercept $\beta 2$ & 0.832 & 0.847 & -0.83 to 2.49 & 0.326 \\
\hline Change in slope $\beta 3$ & 0.068 & 0.046 & -0.02 to 0.16 & 0.134 \\
\hline
\end{tabular}

Fig. 2 Rate of RAR events during the distinct naming strategy period (unshaded area) and the alternative naming strategy period (shaded area). The estimates from the model, and associated confidence intervals are also provided. CI, confidence interval; RAR, retract and reorder. 
week. Temporally, one clinician was found to have only errors in two nonconsecutive weeks out of a total of 25 weeks worked (23 of 25 weeks had no RAR events). The second clinician was found to have errors occurring in 12 out of 60 weeks worked.

\section{Discussion}

Based on a prospective study, we found that the alternative distinct naming strategy did not significantly change the rate of wrong-patient errors as compared with the distinct naming strategy. Although the naming strategy did not result in a significant decrease in wrong-patient errors, we believe that the alternative naming strategy approach is a pragmatic approach that can provide additional safety precautions in newborn and neonatal care settings where patient misidentification rates are known to be historically high. This is because of the ability of alternative naming strategy to potentially distinguish newborns (or newborn and their mothers) during orders, especially in situations where space is limited and names may be truncated. As clinicians increasingly use mobile devices, ${ }^{20}$ misidentification errors are likely to increase. ${ }^{21}$ Although pragmatic, it must be stated that any changes to systems must be made on available evidence, and further, long-term prospective studies are required to evaluate the effectiveness of the alternative distinct naming strategy. ${ }^{22,23}$

We also found that, in our data, the wrong-patient errors were distributed among a very small set of clinicians, highlighting the potential that medication errors are associated with workflows and behaviors of individual clinicians. These high-error clinicians are not unique to our study and are likely to exist in other populations. They can also be plausible targets for clinician-centered interventions and can have as much effect as system-level (e.g., organizational) changes. ${ }^{24-26}$ Targeted mechanisms can include assisting such clinicians through individualized training or clinical decision support. Although this group was too small for a subgroup analysis, it potentially suggests a reason for the lack of overall significant differences.

In addition to the use of distinct naming systems, a federal recommendation from the Joint Commission recommends standardized identification bands like two-site barcodes and also establishing communication tools or alerts among staff for newborns with similar names. ${ }^{8}$ Other than process issues, medication-related adverse events in neonatal settings may occur due to a lack of staff medication safety training and awareness. $^{27}$

These results suggest several avenues for future study. First, a follow-up study with longer duration, multiple locations, or a larger sample size may be powered to detect an effect with the alternative distinct naming strategy. Second, the presence of providers with higher error rates suggests that a targeted approach could be developed and studied as a way to reduce error rates. Finally, the alternative distinct naming strategy maybe especially useful where screen space is limited, such as in mobile interfaces.

\section{Limitations}

There are several limitations for this study. First, this was an exploratory study with retrospective data that was conducted at three hospitals, all part of the same health care system. This was an exploratory study without an a priori hypothesis and as such we did not do a power analysis. Given the smaller size of the hospitals, the volume of orders, and correspondingly, the number of RAR events were low. The study periods-distinct naming and alternative distinct naming-may have been too brief (28 and 34 weeks, respectively) to detect statistically significant differences. We did not account for differences in clinical expertise or role (e.g., resident vs. nurse or practitioner) or individual differences among clinicians who intercepted wrong-patient errors (i.e., who had RAR events). Additionally, given the small number of clinicians included during the considered study periods, the findings are likely generalizable only to similar-sized hospitals. With the culture of patient safety, it is potentially possible that there may have been other patient safety interventions in the considered units during the study period that affected the outcomes. We did not independently validate whether the RAR events were true wrongpatient errors or not. However, previous research has shown the viability of using RAR as a proxy for wrong-patient errors in pediatric and neonatal settings. ${ }^{4,5}$ For the analysis, we did not have data on clinician-type breakdowns of wrong-patent errors. We only used the standard 10-minute interval from the RAR events; errors that were identified by other clinicians or those that were caught later in the medication workflow were not included in the analysis. The errors were clustered around a small number $(n=2)$ clinicians, which may have affected the overall analysis.

\section{Clinical Relevance Statement}

Newborn naming strategies vary across the U.S. hospitals and, as a result, they are vulnerable patient misidentification errors. We developed and evaluated an alternative newborn naming strategy that provides pragmatic advantages for mitigating patient misidentification errors. Based on an exploratory study, we found that errors are potentially clustered around a small set of clinicians, so targeted mechanisms can potentially be developed to assist these clinicians. Future prospective studies on the use of the alternative distinct naming strategy are required to establish its viability in clinical settings.

\section{Multiple Choice Questions}

1. Retract-and-reorder (RAR) events are a proxy for what type of error?

a. Laboratory errors

b. Adverse drug reactions

c. Wrong patient errors

d. Physician fatigue errors

Correct Answer: The correct answer is option c. A RAR event is defined as any order-medication, laboratory, imaging, or general care orders-placed and retracted on a patient within 10 minutes, and then reordered by 
the same clinician on a different patient within the next 10 minutes. RAR is also recommended by the National Quality Forum and the Office of the National Coordinator for Health Information Technology as a metric for wrongpatient errors. The NQF determined that RAR events are "an outcome measure because while the error did not actually reach the patient, a wrong-patient RAR in the EHR is still a medical error." The RAR measure was originally developed by Adelman and colleagues and was found to have a $76.2 \%$ positive predictive value for identifying a wrong-patient order.

2. What is a benefit of the alternate distinct naming strategy over the distinct naming strategy?

a. Twins no longer have the same name.

b. The newborn's date of birth is included.

c. Names can be entered earlier into the medical record.

$\mathrm{d}$. The newborn's gender is more visible in a header.

Correct Answer: The correct answer is option d. Examples of the naming strategies can be seen in -Fig. 1. The alternative distinct naming strategy uses the newborn's gender as a prefix to the mother's first name in the following manner: "Last Name, GenderFirst Name" (e.g., "Washington, BoyMartha”).

In most EHRs, headers display patient names in the format "Last Name, First Name," but have limits on the number of characters that can be displayed. For example, in the Epic EHR (Verona), there is a spatial limit on the tab header, allowing approximately 17 characters to be displayed. As a result, when using the distinct newborn strategy, newborns with long last names can have their gender information cut-off and not show up on the header. For example, "Washington, Marthasboy" will be displayed in the header as "Washington, Marth." This truncation becomes particularly problematic as a clinician caring for a newborn may need to frequently access both the newborn's and the mother's charts, increasing the potential for wrong-patient errors. To avoid patient misidentification errors, we developed and implemented an alternative distinct naming strategy within our institution's EHR. With this strategy, the appearance of the mother's name and the newborns name in the header should be more distinct (e.g., "Washington, Marth" for the mother's header and "Washington, BoyMa” for her son's header).

With the alternative naming strategy, the likelihood of a newborn's gender being truncated in a header is possible only for very long last names.

Authors' Contributions

E.P., M.L., J.A., and T.G.K. conceived the study. E.P. and T.G. $K$. conducted the data analysis; all authors reviewed the analysis and were involved in the writing of the manuscript.

Protection of Human and Animal Subjects

The study was performed in compliance with the World Medical Association Declaration of Helsinki on Ethical Principles for Medical Research Involving Human Subjects and was reviewed by Washington University Institutional Review Board.

\section{Funding}

This research was supported in part by a grant (No. R21HS025443) from the Agency for Healthcare Research and Quality (AHRQ). The content is sole responsibility of the authors and does not necessarily represent the official views of the AHRQ.

Conflict of Interest

None declared.

\section{References}

1 Martin JA, Hamilton BE, Osterman MJK, Curtin SC, Matthews TJ. Births: final data for 2013. Natl Vital Stat Rep 2015;64(01):1-65

2 Suresh G, Horbar JD, Plsek P, et al. Voluntary anonymous reporting of medical errors for neonatal intensive care. Pediatrics 2004;113 (06):1609-1618

3 Gray JE, Suresh G, Ursprung R, et al. Patient misidentification in the neonatal intensive care unit: quantification of risk. Pediatrics 2006;117(01):e43-e47

4 Adelman JS, Aschner JL, Schechter CB, et al. Evaluating serial strategies for preventing wrong-patient orders in the NICU. Pediatrics 2017;139(05):e1-e7

5 Adelman J, Aschner J, Schechter C, et al. Use of temporary names for newborns and associated risks. Pediatrics 2015;136(02): 327-333

6 Wang J, Liang H, Kang H, Gong Y. Understanding health information technology induced medication safety events by two conceptual frameworks. Appl Clin Inform 2019;10(01):158-167

7 YorkJB, Cardoso MZ, Azuma DS, Beam KS, Binney GG Jr, Weingart SN. Computerized physician order entry in the neonatal intensive care unit: a narrative review. Appl Clin Inform 2019;10(03):487-494

8 Commission J. Temporary names put newborns at risk. Quick Safety 2015;17:1-2

9 Adelman JS, Aschner JL, Schechter CB, et al. Babyboy/babygirl: a national survey on the use of temporary, nondistinct naming conventions for newborns in neonatal intensive care units. Clin Pediatr (Phila) 2017;56(12):1157-1159

10 National Quality Forum. Patient safety 2015. Available at: https:// www.qualityforum.org/Publications/2016/02/Patient_Safety_2015_ Final_Report.aspx. Accessed February 13, 2020

11 Office of the National Coordinator for Health Information Technology (ONC). Self-assessment patient identification: General Instructions for the SAFER Self-Assessment Guides. Available at: https:// www.healthit.gov/sites/default/files/safer_patient_identification. pdf. Accessed February 13, 2020

12 Adelman JS, Kalkut GE, Schechter CB, et al. Understanding and preventing wrong-patient electronic orders: a randomized controlled trial. J Am Med Inform Assoc 2013;20(02):305-310

13 Kannampallil TG, Manning JD, Chestek DW, et al. Effect of number of open charts on intercepted wrong-patient medication orders in an emergency department. J Am Med Inform Assoc 2018;25(06): 739-743

14 Green RA, Hripcsak G, Salmasian H, et al. Intercepting wrongpatient orders in a computerized provider order entry system. Ann Emerg Med 2015;65(06):679-686.e1

15 Feldstein AC, Smith DH, Perrin N, et al. Reducing warfarin medication interactions: an interrupted time series evaluation. Arch Intern Med 2006;166(09):1009-1015

16 Smith DH, Perrin N, Feldstein A, et al. The impact of prescribing safety alerts for elderly persons in an electronic medical record: an interrupted time series evaluation. Arch Intern Med 2006;166 (10):1098-1104 
17 Mullan PC, Scott S, Chamberlain JM, et al. Decreasing blood culture contaminants in a pediatric emergency department: an interrupted time series analysis. Pediatr Qual Saf 2018;3(05):e104

18 Wagner AK, Soumerai SB, Zhang F, Ross-Degnan D. Segmented regression analysis of interrupted time series studies in medication use research. J Clin Pharm Ther 2002;27(04):299-309

19 Team RC. R: a language and environment for statistical computing. Available at: https://www.gbif.org/tool/81287/r-a-language-andenvironment-for-statistical-computing. Accessed February 13, 2020

20 Anand V, McKee S, Dugan TM, Downs SM. Leveraging electronic tablets for general pediatric care: a pilot study. Appl Clin Inform 2015;6(01):1-15

21 Warm D, Edwards P. Classifying health information technology patient safety related incidents - an approach used in Wales. Appl Clin Inform 2012;3(02):248-257

22 Freed GL. When new standards to improve safety do not actually improve safety. JAMA Pediatr 2019;173(10):921-922
23 Wachter RM, Murray SG, Adler-Milstein J. Restricting the number of open patient records in the electronic health record: is the record half open or half closed? JAMA 2019;321(18):1771-1773

24 Conn VS, Ruppar TM, Enriquez M, Cooper PS, Chan KC. Healthcare provider targeted interventions to improve medication adherence: systematic review and meta-analysis. Int J Clin Pract 2015; 69(08):889-899

25 Kingdon C, Downe S, Betran AP. Non-clinical interventions to reduce unnecessary caesarean section targeted at organisations, facilities and systems: systematic review of qualitative studies. PLoS One 2018;13(09):e0203274

26 Meeker D, Linder JA, Fox CR, et al. Effect of behavioral interventions on inappropriate antibiotic prescribing among primary care practices: a randomized clinical trial. JAMA 2016;315(06): 562-570

27 Kunac DL, Reith DM. Identification of priorities for medication safety in neonatal intensive care. Drug Saf 2005;28(03):251-261 